\title{
Numerical Simulation of Vibration Deflection Effects on the Energy Efficiency of Ultrasonic Transducer for Sonochemistry
}

\author{
Chang Ge \\ Department of Electrical and Computer Engineering, University of Alberta, 2204 111A Street NW, Edmonton, AB, Canada T6J 4V6 \\ Correspondence should be addressed to Chang Ge; cge@ualberta.ca
}

Received 11 September 2014; Accepted 16 November 2014

Academic Editor: Song Cen

Copyright (C) 2015 Chang Ge. This is an open access article distributed under the Creative Commons Attribution License, which permits unrestricted use, distribution, and reproduction in any medium, provided the original work is properly cited.

\begin{abstract}
The energy efficiency of ultrasound transducers for sonochemistry applications was studied from the point of view of vibration deflection effects of different transducer structures, including the configurations of rectangular plate, cylindrical shell, triangular strip array, rectangular strip array, and pentagonal strip array. It was found that the deflections are not uniform over the transducer surface with the maximum deflection locating at the geometry center of transducers. The simulated maximum deflection of rectangular plate results in the value of $2.5 \times 10^{-16}$, which is larger than the maximum deflection of cylindrical shell with the value of $2 \times 10^{-16}$. This finding suggests that the envelope deflection volume of two transducers has significant difference, which has been neglected in the design and selection of ultrasound transducer. For this reason, a quantitative index involving the envelope deflection volume was proposed to compare the efficiency of ultrasound transducers. Based on the simulation results, the triangular strip array transducer structure with higher ultrasound generation efficiency was proposed, which was also theoretically validated by the MATLAB simulation.
\end{abstract}

\section{Introduction}

The propagation of ultrasound waves through liquids at frequencies ranging from $20 \mathrm{kHz}$ to a few megahertz results in cavitation, which causes various physical and chemical effects. During the 1920s, the acoustic waves were used to generate cavitations that were found to significantly change the reaction rate. In the 1960 s, the commercial ultrasonic devices began to be applied for chemistry reaction, termed as sonochemistry. In the recent years, ultrasonic irradiation attracted researchers to incorporate it in many new areas such as manufacturing of nanostructured materials, food industry, processing of biomass, and sonochemical degradation of pollutant materials and hazardous chemicals [1].

Biofuel is a renewable, clean, and alternative fuel having potential application in the future. Ultrasonic irradiation has been proven to be successfully used to increase the mass transfer rate during the biodiesel reaction, showing promising results on the reaction variables compared with the results of conventional process $[1,2]$. Luo et al. concluded that the ultrasonic cavitation can break the immiscible binary reactants, oils and alcohols, into tiny emulsion and therefore enhance the hydrolysis, esterification, and transesterification reactions in biodiesel synthesis [3].

Several ultrasound parameters, such as its frequency, intensity, duty cycle, and duration of irradiation, determine the potential effects on chemical reaction. Among these factors, the frequency and intensity of ultrasound have a significant influence on the efficiency of sonochemistry process [47]. Tsochatzidis et al. found that the influence of ultrasound power input increased bubble velocity quasilinearly and has more complex effect on bubbles' diameter [4]. Entezari and Kruus investigated the effects of temperature and intensity on the rate of sonochemical oxidation of aqueous potassium iodide with irradiation at frequencies of 20 and $900 \mathrm{kHz}$ [5]. Merouani et al. proposed a model to investigate the effects of ultrasound frequency and acoustic amplitude on the size of sonochemically active bubble [6]. The observation on acoustic cavitation phenomena at $1 \mathrm{MHz}$ ultrasound showed that cavitation activity was strongly linked to the occurrence of fast-moving bubbles. It was shown that bubble activity can be stabilized and enhanced by the use of pulsed ultrasound by 

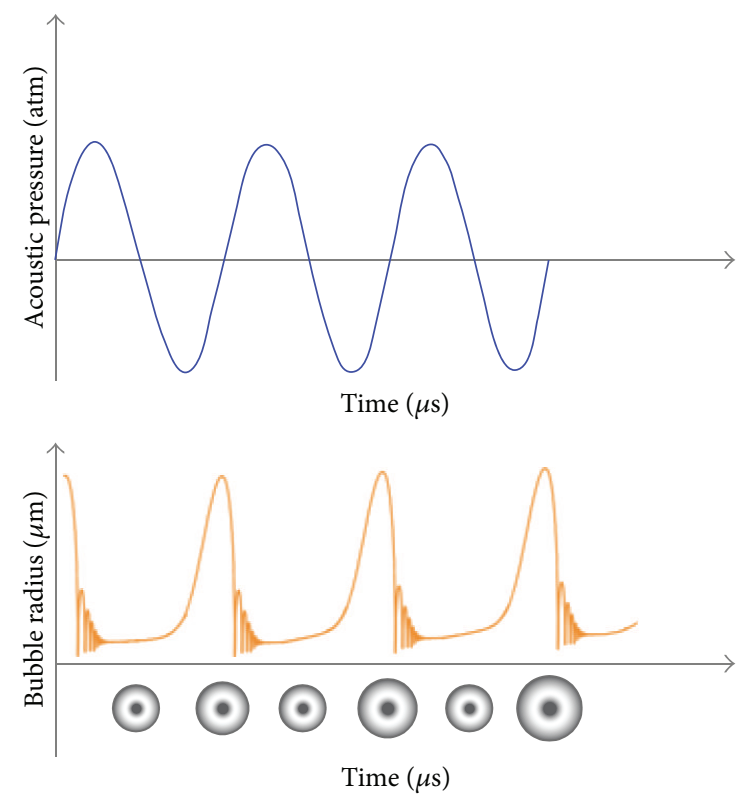

FIGURE 1: Illustration of bubble oscillation corresponding to pressure antinodes (redrawn from [9]).

conserving and recycling active bubbles between subsequent pulsing cycles [7].

In the published literatures, most of them are aimed at the effect of frequency of ultrasound transducer on the efficiency of sonochemistry whereas few of them discussed the effect of transducer vibrating amplitude. Vibration of ultrasound transducer is usually forced. The amplitude of wave generated is directly related to the deflection of the transducer in vibration. In addition, amplitude is directly related to the intensity of the ultrasound generated. In this paper, a study was carried out on the effect of transducer structure on vibrating amplitude. Calculation was carried out, and theoretical optimization for a more efficient structure from amplitude point of view was proposed. The effectiveness of the structure was validated by MATLAB simulations.

\section{Quantification of Ultrasound Efficiency}

2.1. Ultrasonication Mechanism. Sonication is the act of applying sound energy to agitate particles in a sample by the cavitation propagation, for various purposes such as cleaning, disintegration, dispersion, and sonochemistry. Cavitation can be considered as one of the methods of introducing discrete energy input, which is activated by ultrasound transducers, where the energy dissipated per unit volume is few orders of magnitude higher than the conventional processes.

The cavitational activation in heterogeneous systems mainly results from a consequence of the mechanical effects. Cavitation can be classified as transient cavitation and stable cavitation. Generation of transient or stable cavitation usually depends on the set of operating parameters and constitution of the liquid medium. Transient cavitation is generally due to gaseous or vapor filled cavities, which are believed to be produced at ultrasonic intensity greater than $10 \mathrm{~W} / \mathrm{cm}^{2}$. The bubble formed in stable cavitation contains gas at ultrasonic intensity in the range of $1-3 \mathrm{~W} / \mathrm{cm}^{2}$ [8].

The dynamic change of bubble in accordance with ultrasonic waves is shown as in Figure 1. The standing acoustic wave causes the bubbles to quickly coalesce into a single radially oscillating bubble. Once a single bubble is stabilized in the pressure antinode of the standing wave, it can be made to emit pulses of waves by driving the bubble into highly nonlinear oscillations. The collapsed bubble expands due to high internal pressure and experiences a diminishing effect until the high pressure antinode returns to the center of the vessel. The bubble continues to occupy more or less the same space due to the acoustic radiation force [9].

2.2. Bubble Collapse Energy. In case the condition of the incompressibility of the medium is relaxed and the external pressure is considered as the time dependent pressure $p(t)$, the collapse pressure of cavity bubble is calculated using the formula [10]

$$
p_{\text {collapse }}=\left(p_{0}+\frac{2 \sigma}{R_{0}}\right) \times\left(\frac{R_{0}}{R}\right)^{3 \gamma}-\frac{2 \sigma}{R}-\frac{4 \mu}{R}\left(\frac{d R}{d t}\right),
$$

where $p_{0}$ is the ambient pressure, $R_{0}$ is the initial radius of cavity, $\sigma$ is the surface tension of liquid, and $\mu$ is the viscosity. It is suggested that the bubble collapse pressure decreases with an increase in the intensity of the ultrasound. The maximum cavity size decreases with increasing frequency, but the collapse of the cavity is very rapid thereby leading to an increase in the magnitude of the collapse pressure [10]. 


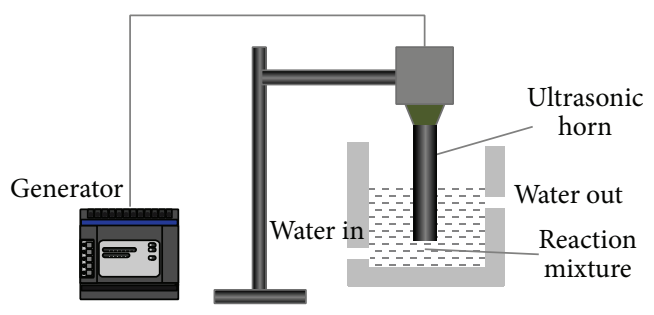

(a)

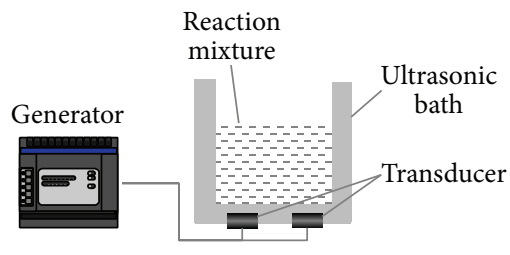

(b)

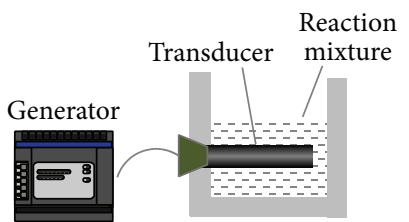

(c)

FIGURE 2: Three configurations of sonochemical reactor.

The pressure and temperature inside the bubble at any instant during adiabatic phase can be calculated from the bubble size, using the adiabatic law [11]:

$$
\begin{gathered}
p=\left[p_{v}+p_{g 0}\left(\frac{R_{0}}{R_{\max }}\right)^{3}\right]\left(\frac{R_{\max }}{R}\right)^{3 \gamma}, \\
T=T_{\infty}\left(\frac{R_{\max }}{R}\right)^{3(\gamma-1)},
\end{gathered}
$$

where $p_{v}$ is the saturated vapor pressure, $p_{g 0}=p_{\infty}+$ $\left(2 \sigma / R_{0}\right)-p_{v}$ is the gas pressure in the bubble at its ambient state $\left(R=R_{0}\right), R_{0}$ is the ambient bubble radius, $T_{\infty}$ is the bulk liquid temperature, $R_{\max }$ is the maximum radius of the bubble, and $c$ is the ratio of specific heat capacities $\left(c_{p} / c_{v}\right)$ of the vapor/gas mixture. At the end of the bubble collapse, the bubble radius becomes the minimum $R_{\min }$; the maximum internal temperature $\left(T_{\max }\right)$ and pressure $\left(p_{\max }\right)$ will result.

When higher acoustic amplitudes are applied, the bubble results in higher compression ratios $R_{\max } / R_{\min }$ and higher $T_{\max }$ and $p_{\max }$. Thus a higher energy is generated in the bubble at the collapse. Merouani et al. reported the power increased linearly with increasing acoustic amplitude from 1.5 to $3 \mathrm{~atm}$, which was described as Power $(\mathrm{mW})=5.1557 P_{A}(\mathrm{~atm})-$ 6.7369, in which $P_{A}$ is the acoustic amplitude (atm) [11].

The ultrasonic power dissipated into a liquid was calculated by the following equation [12]:

$$
\text { Power }(\mathrm{W})=c_{p} M \frac{d T}{d t}
$$

where $c_{p}$ is the heat capacity of water $\left(4.2 \mathrm{Jg}^{-1}\right)$ and $M$ is the mass of water $(\mathrm{g}) .(d T / d t)$ is the temperature rise per second.

\subsection{Actual Sonoreactor Efficiency. Various types of sono-} chemical reactor have been designed in engineering applications. The common used transducers are of disc shape, cylindrical shape, and horn shape.

Csoka et al. introduced three sonochemical reactor configurations which consist of an ultrasonic horn, an ultrasonic cleaning tank type reactor, and an ultrasonic longitudinal horn, as shown in Figure 2. They observed that the actual power dissipation for horn at an operating capacity of $300 \mathrm{~mL}$ is $29.3 \mathrm{~W}$ giving an energy efficiency of $12.7 \%$, for bath at an operating capacity of $3.3 \mathrm{~L}$ is $224 \mathrm{~W}$ giving an energy efficiency of $24 \%$, and for longitudinal horn at an operating

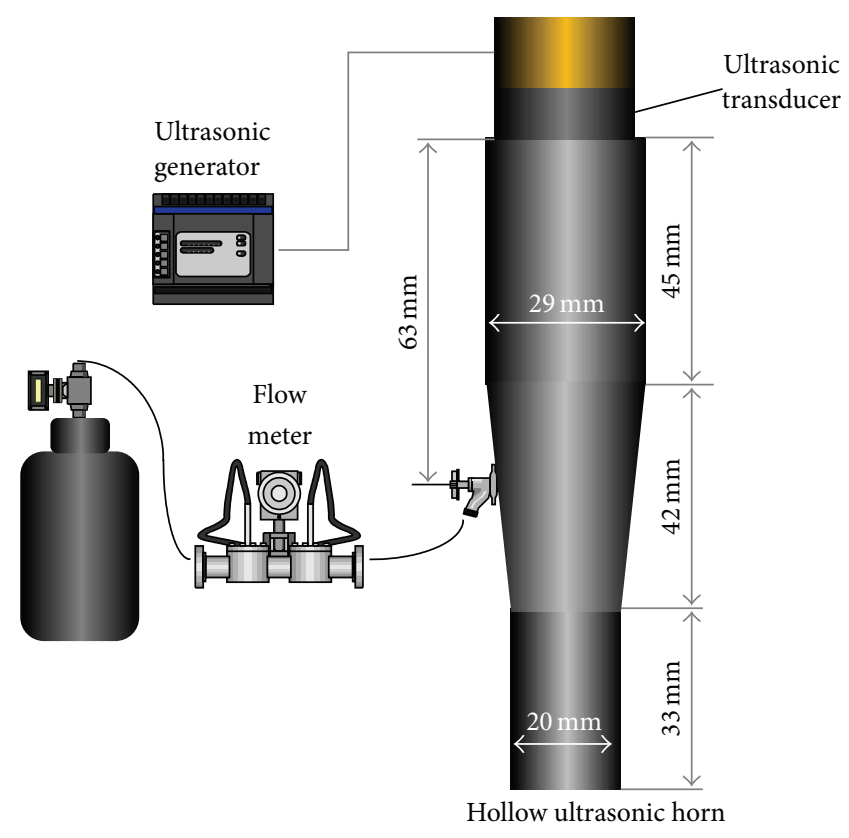

FIGURE 3: Sonochemical reactor with a hollow cylindrical ultrasonic horn.

capacity of $9.5 \mathrm{~L}$ is $79.9 \mathrm{~W}$ giving an energy efficiency of $59.2 \%$ [13].

Makuta et al. developed a novel and simple sonochemical reactor with a hollow cylindrical ultrasonic horn, as shown in Figure 3 [14]. The relationship between the electrical power input $\left(P_{\text {in }}, \mathrm{W}\right)$ to the transducer and the peak-to-peak oscillatory displacement $(y, \mu \mathrm{m})$ of the smaller end can be expressed by the following approximate equation [14]:

$$
y=53.24\left(1-e^{-0.01785 P_{\text {in }}}\right) \text {. }
$$

Asakura et al. found that the sonochemical efficiency (SE) depended on the frequency and liquid height; that is, between 45 and $490 \mathrm{kHz}$, the peak of the SE value increases monotonically with the logarithm of the frequency, and the liquid height yielding the highest SE is approximately 15 times the wavelength [15].

These research results demonstrate that the energy efficiency of sonoreactor is strongly dependent on the shape of ultrasound transducer and arrangement position. The energy efficiency of a vertical immersed cylindrical sonoreactor is about $12 \%$, but a longitudinal cylindrical sonoreactor results 


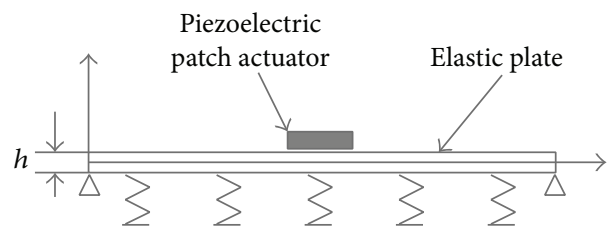

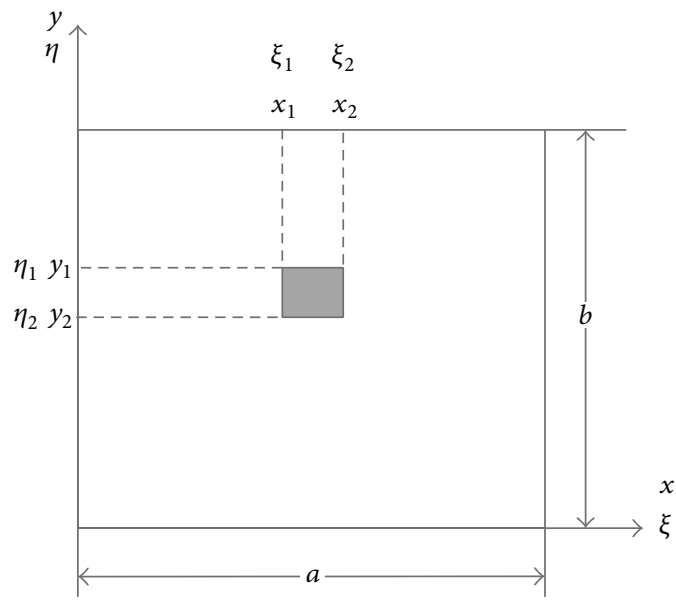

(a)

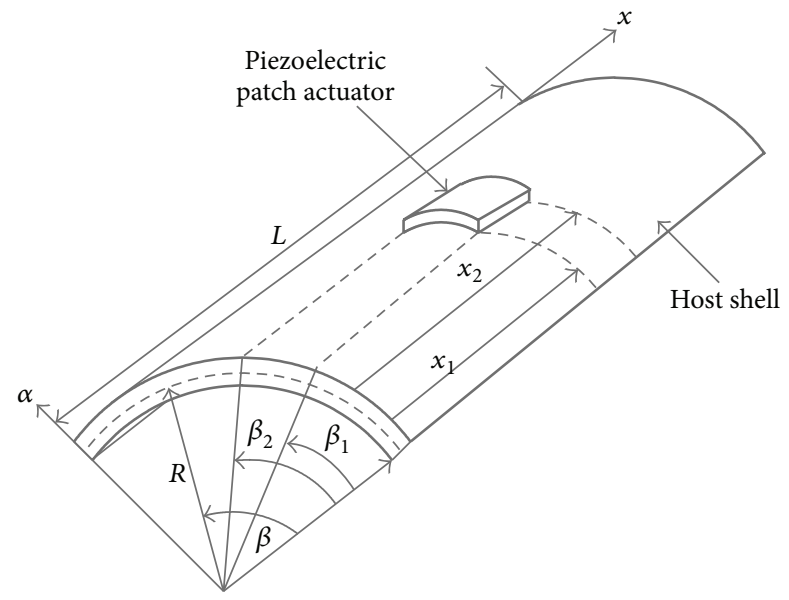

(b)

FIGURE 4: Illustrations of mechanics model of a rectangular plate transducer and a cylindrical transducer with piezoelectric patch actuator: (a) rectangular plate transducer and (b) cylindrical transducer.

in energy efficiency as high as $60 \%$. The plate ultrasound transducers provide energy efficiency twice that for a vertical immersed cylindrical reactor. This inspires the investigation into the intrinsic mechanism of vibration behavior and its effect on the energy efficiency.

\section{Vibration Deflection of Ultrasound Transducers}

3.1. Governing Equations. Equations describing vibration deflection for different ultrasound transducer structures are provided in literature [16]. The two most popular transducer structures, rectangular plate and cylindrical shells, are studied. Settings for analysis of the two structures are shown in Figures 4(a) and 4(b), respectively. Matching layer is treated as a load for piezoelectric actuator.

Governing equations of deflection for a rectangular plate transducer and a cylindrical shell transducer are [16]

$$
\begin{aligned}
& w_{\text {rectangular }}=\sum_{n=1}^{\infty} \sum_{m=1}^{\infty} \frac{16 M_{0}}{a^{2} \rho h} * \frac{\sin \omega t}{\omega_{m n}^{2}-\omega^{2}} *\left(\frac{m}{n}+\frac{n \beta^{2}}{m}\right) \\
& * \sin m \pi \xi_{0} * \sin n \pi \eta_{0} * \sin m \pi r_{\xi} \\
& * \sin n \pi r_{\eta} * \sin m \pi \xi * \sin n \pi \eta, \\
& w_{\text {cylindrical }}=\sum_{m=1}^{\infty} \sum_{n=1}^{\infty} \frac{4 B_{m n} \sin n \pi \theta_{0} \sin m \pi \xi_{0}}{\omega_{m n}^{2}-\omega^{2}} \\
& \\
& \times \sin m \pi \xi \sin n \pi \theta e^{j \omega t} .
\end{aligned}
$$

Equation (5) is for the rectangular plate. Equation (6) is for the cylindrical shell. $\omega_{m n}^{2}$ is the square value of natural frequency for a transducer structure. $M_{0}$ and $B_{m n}$ are the constants related to voltage and stress which resulted from reverse piezoelectric effect. $\xi$, $\eta$, and $\theta$ are all normalized coordinates of an arbitrary point on the transducer surface plane. Details for constant expression can be found in the literature.

Calculations in MATLAB for (5) and (6) were carried out in this paper. For the rectangular plate transducer, the width of the plate was set to $19 \mathrm{~mm}$. The height of the plate was set to $20 \mathrm{~mm}$. The thickness of the actuator was set to $0.6 \mathrm{~mm}$. The actuator was considered to cover the entire load surface, which is represented by the elastic plate in Figure 4(a) and the host shell in Figure 4(b). The thickness of the load was set to $0.25 \mathrm{~mm}$. For the cylindrical shell transducer, the height of the transducers was set to $20 \mathrm{~mm}$. The diameter was set to $19 \mathrm{~mm}$. The cutout angle was set to $\pi$. Other settings were the same as the ones for the rectangular plate transducer. The decisive factors of the surface deflection in (5) and (6) are the series of $m$ and $n$. Equations (5) and (6) can be reshaped into

$$
\begin{aligned}
& W_{\text {rectangular }} \\
& \begin{array}{c}
\sum_{m=1}^{\infty} \sum_{n=1}^{\infty}\left(\frac{16 M}{\rho h a^{2}}\left(\frac{m}{n}+\frac{n}{m} \beta^{2}\right)\left(\sin \frac{n \pi}{2}\right)^{2}\left(\sin \frac{m \pi}{2}\right)^{2}\right. \\
\times \sin m \pi \xi * \sin n \pi \eta)\left(\omega_{m n}^{2}-\omega^{2}\right)^{-1}
\end{array}
\end{aligned}
$$




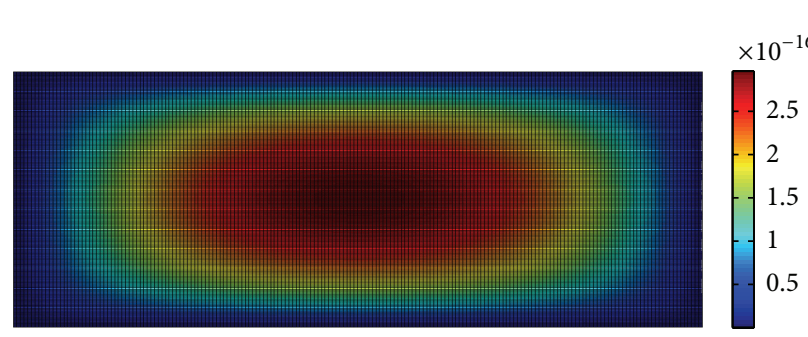

(a)

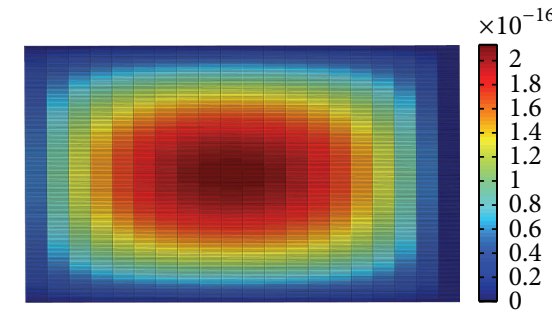

(b)

FIGURE 5: Simulation results of surface deflection: (a) rectangular plate transducer and (b) cylindrical shell transducer.

$$
\begin{aligned}
& W_{\text {shell }} \\
& \begin{array}{c}
\sum_{m=1}^{\infty} \sum_{n=1}^{\infty}\left(\frac{16 M}{\rho h a^{2}}\left(\frac{m}{n}+\frac{n 4}{m \pi^{2}} \beta^{2}\right)\left(\sin \frac{n \pi}{2}\right)^{2}\left(\sin \frac{m \pi}{2}\right)^{2}\right. \\
\left.\quad \times \sin m \pi \xi \sin n \pi \theta e^{j \omega t}\right)\left(\omega_{m n}^{2}-\omega^{2}\right)^{-1}
\end{array}
\end{aligned}
$$

Since the cylindrical transducer and rectangular plate transducer are made from same material in this paper, for a more general conclusion, $16 \mathrm{M} / \rho h \mathrm{a}^{2}$ in (7) was simplified to a constant of 1 in this paper.

Figure 5 illustrates the calculation results of (5) and (6). The distribution over the cylindrical shell was planarized before illustration. Obvious differences between the deflection of the rectangular plate and the cylindrical shell can be observed. The deflection of rectangular transducer shows racetrack pattern, while cylindrical transducer shows ellipse pattern. The cover area of the deflection on rectangular transducer is larger than that of cylindrical transducer.

The deflections are not uniform over the structure surface. The maximum deflection locates at the geometry center of transducers and then gradually reduces to the minimum at the edge of transducer. In addition, the simulated maximum deflection of rectangular plate results in the value of $2.5 \times$ $10^{-16}$, which is larger than the maximum deflection of cylindrical shell with the value of $2 \times 10^{-16}$. This finding suggests that the envelope volume of the transducer's surface deflection has very large difference, which is neglected in the design and selection of ultrasound transducer. For this reason, the efficiency comparison for different transducer structures should be carried out in view of the envelope deflection over the structure surface.

3.2. Deflection Volume of Transducer. To compare envelope deflection effects of transducer structure on the ultrasound vibration intensity, the surface deflection integrals of (5) and (6) are required. The integrals represent the global effect of vibration deflection over the transducer surface, which is quantitatively described as deflection volume.
The integrals of (5) and (6) are obtained as follows:

$$
\begin{aligned}
& W_{\text {rectangular }} \\
& =\sum_{m=1}^{\infty} \sum_{n=1}^{\infty}\left(\frac{16 M}{\rho h a^{2}}\left(\frac{m}{n}+\frac{n}{m} \beta^{2}\right)\left(\sin \frac{n \pi}{2}\right)^{2}\left(\sin \frac{m \pi}{2}\right)^{2}\right. \\
& \left.\times \frac{\left(1-(-1)^{m}\right)}{m} \frac{\left(1-(-1)^{n}\right)}{n}\right) \\
& \times\left(\omega_{m n}^{2}-\omega^{2}\right)^{-1}, \\
& W_{\text {shell }} \\
& =\sum_{m=1}^{\infty} \sum_{n=1}^{\infty}\left(\frac{16 M}{\rho h a^{2}}\left(\frac{m}{n}+\frac{n 4}{m \pi^{2}} \beta^{2}\right)\left(\sin \frac{n \pi}{2}\right)^{2}\left(\sin \frac{m \pi}{2}\right)^{2}\right. \\
& \left.\times \frac{\left(1-(-1)^{m}\right)}{m} \frac{\left(1-(-1)^{n}\right)}{n}\right) \\
& \times\left(\omega_{m n}^{2}-\omega^{2}\right)^{-1} .
\end{aligned}
$$

In both calculations, it is assumed that piezoelectric actuator covers the whole load surface, which is represented by the elastic plate in Figure 4(a) and the host shell in Figure 4(b).

\section{Theoretical Efficiency Analysis of Ultrasound Transducer}

The acoustic intensity of ultrasound transducer has a positive relationship with the deflection volume, because the acoustic intensity has a positive relationship with the ultrasound amplitude, which has a positive relationship with the deflection [17]. Since the actuator forces leading to displacement are linearly proportional to the voltage applied on the transducer, the ultrasound transformation efficiency from electrical energy to mechanical vibration can be defined as the deflection volume per unit of voltage, which is stated as

$$
\varepsilon=\frac{W}{V},
$$

where $\varepsilon$ is the energy efficiency, $W$ is the vibration volume, and $V$ is the electrical voltage applied on the transducer. For 


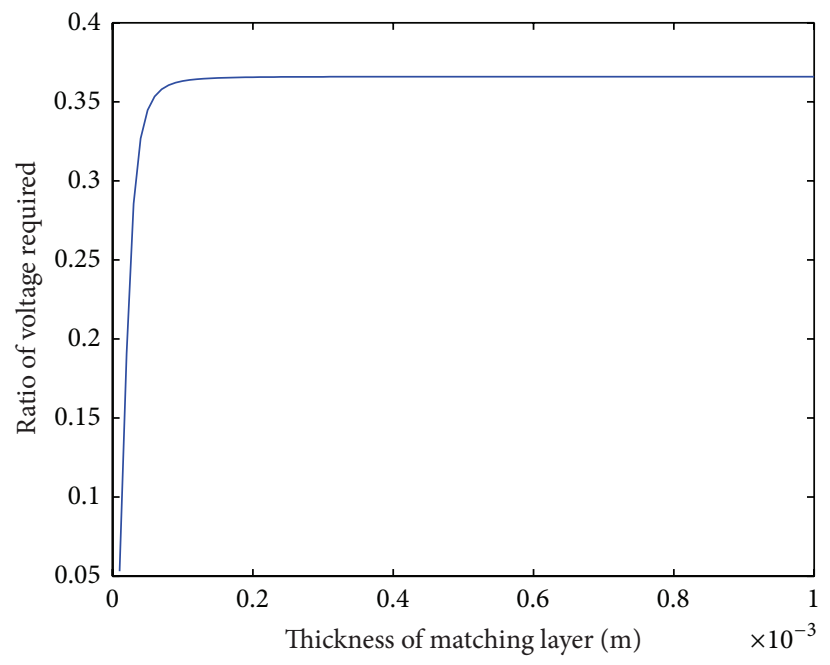

FIGURE 6: Ratio of voltage (rectangular/cylindrical) required for the equal deflection volume.

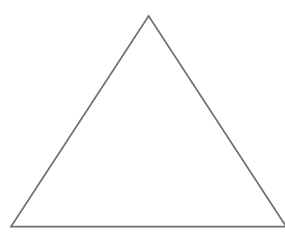

(a) Triangle

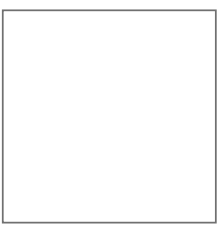

(b) Quadrangle

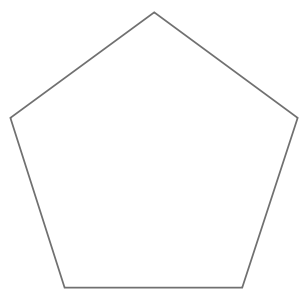

(c) Pentagon

FIgURE 7: Different layouts of ultrasound transducer with various configurations of plate actuators.

the same expected deflection volume, higher electrical voltage applied means lower energy efficiency of an ultrasound transducer. Hence, the ratio of voltage required for the same deflection volume can be adopted as an index to compare the energy efficiency of different transducers.

In order to compare the efficiency between a rectangular plate transducer and a cylindrical transducer, the ratio of voltage to get the same deflection volume was calculated, the result of which was shown in Figure 6. In the simulation process, the thickness of matching layer and the actuator was identical for two transducers. The height was set to $20 \mathrm{~mm}$ for both rectangular plate and cylindrical transducer. The width of the rectangular plate and the diameter of the cylindrical shell were set to $19 \mathrm{~mm}$. The frequency of the ultrasound wave generated was $1.5 \mathrm{MHz}$. Figure 6 shows the simulation results over a wide range of matching layer thicknesses. It can be observed that there is very little variation in the deflection volume ratio when matching layer thickness is over $0.1 \mathrm{~mm}$. The applied voltage for the rectangular plate transducer is only $35 \%$ of that for the cylindrical transducer. These results indicate that the efficiency of rectangular plate transducer is higher than cylindrical shell transducer. In addition, thinner matching layer represents shorter ultrasound wavelength and higher ultrasound frequency; it can also be inferred that the transducer efficiency will increase when the actuator frequency increases.

Uniform ultrasound distribution is a merit of cylindrical transducer. Simply replacing cylindrical transducer with a pair of rectangular plates can lead to negative impact on uniformity of ultrasound distribution. Ultrasound arrays consisting of rectangular strip transducers are trade-offs between the efficiency and the uniformity. They have higher efficiency compared with a cylindrical transducer and less impact on the uniformity of ultrasound distribution compared with a pair of rectangular plates.

In this paper, three ultrasound array structures were investigated, the top views of which are shown in Figures $7(\mathrm{a}) \sim 7$ (c). If $2 \mathrm{U}$ is needed for a cylindrical transducer to reach expected deflection volume, the voltages needed for three arrays shown in Figures $7(\mathrm{a}) \sim 7(\mathrm{c})$ are $1.05 \mathrm{U}, 1.40 \mathrm{U}$, and $1.75 \mathrm{U}$, respectively. It can be inferred that only the arrangements in Figures $7(\mathrm{a}) \sim 7(\mathrm{c})$ meet the requirement of higher efficiency.

Simulations of ultrasound distribution from all three possible array structures were carried out. All simulations were run in the MATLAB tool named "K-wave." A sine function with the amplitude of $2 \mathrm{~Pa}$ and frequency of $1.5 \mathrm{MHz}$ was used in all three simulations. Global environment settings including attenuation coefficient, propagation speed, 


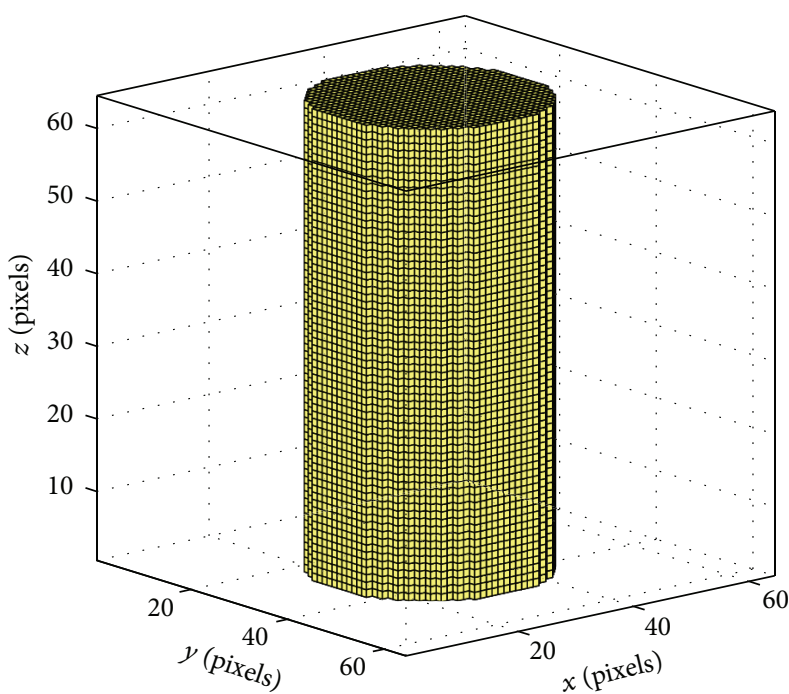

(a)

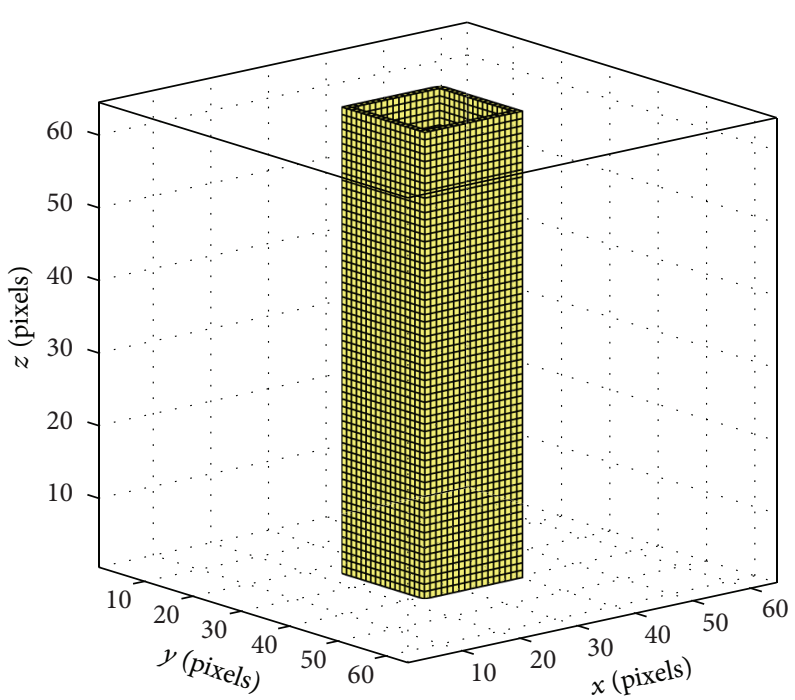

(c)

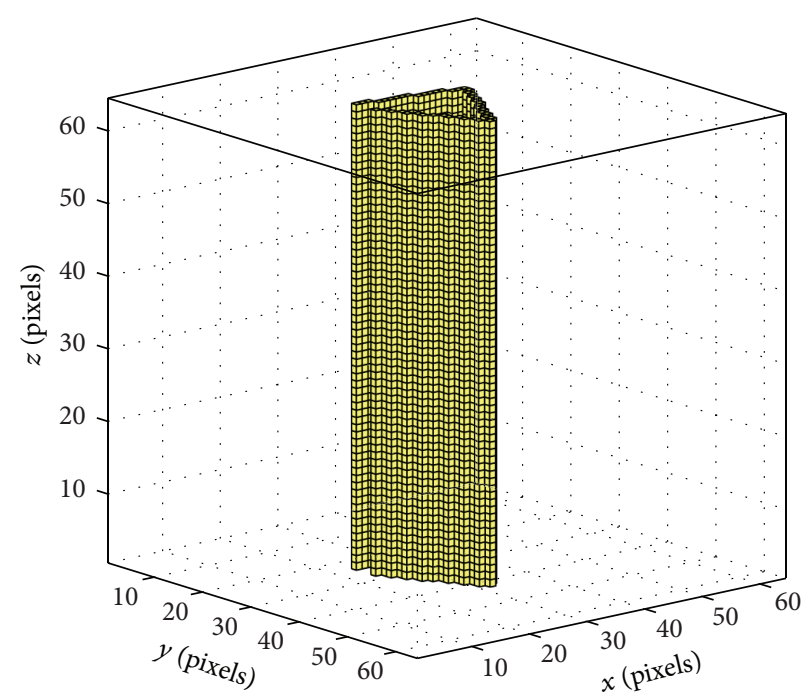

(b)

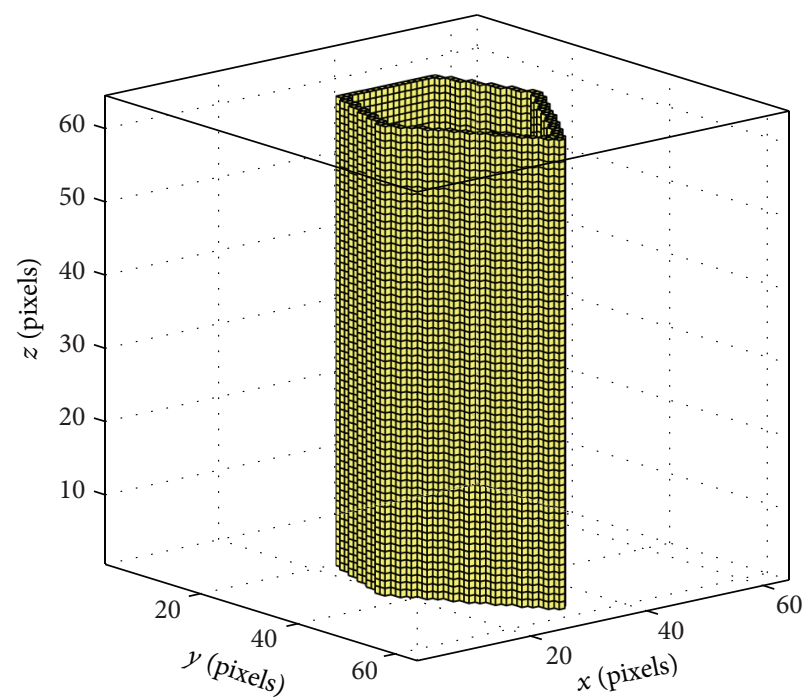

(d)

FIGURE 8: Implementation of different structures in MATLAB simulation: (a) cylindrical transducer, (b) triangular array, (c) rectangular array, and (d) pentagonal array.

medium density, and the position of sensors collecting data were identical for all simulations as well. Figure 8 illustrates the implementation of structures in MATLAB.

Figure 9 illustrates ultrasound distribution simulation result for a cylindrical transducer and the transducer arrays consisting of rectangular plates. The simulation results were planarized for comparison. It can be observed that the triangular array and the pentagonal array have the ultrasound distribution similar to the cylindrical transducer, and the uniformity of ultrasound field from a quadrangular array is worse. The ultrasound distribution from the triangular array is more symmetric than the distribution from the pentagonal array. In addition, it can be inferred from Figure 9 that the maximum ultrasound amplitude of the cylindrical transducer is only around $0.9 \mathrm{~Pa}$, while the maximum ultrasound amplitudes of arrays in all three possible structures are over
1.6 Pa. This fact validates the previous conclusion about a higher efficiency of arrays with rectangular plate elements. Therefore, the triangular array should be selected as the substitute in applications like biofuel productions, in which a higher efficiency of ultrasound transducer is expected.

\section{Conclusions}

In this paper, transducer arrays made by rectangular plate elements are found to have higher efficiency than cylindrical transducers in ultrasound generating. When the cylindrical transducer and the arrays generate ultrasound waves with the same amplitude, lower voltage is required for the arrays. Three arrangements were proposed for the arrays, among which the triangular array structure was found to be a better 


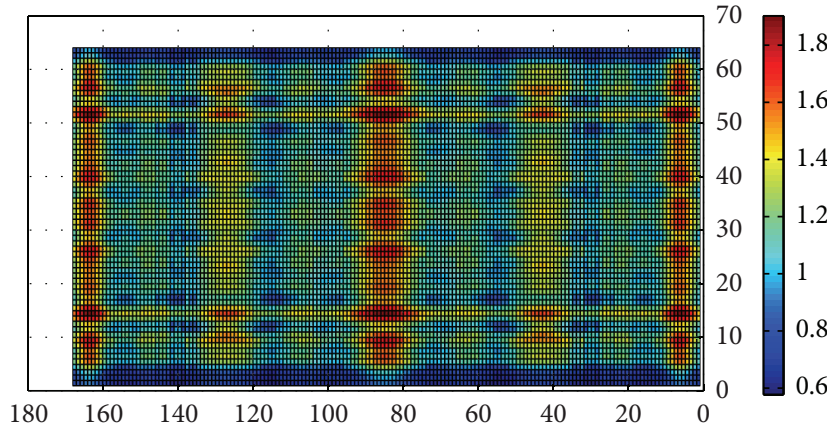

(a)

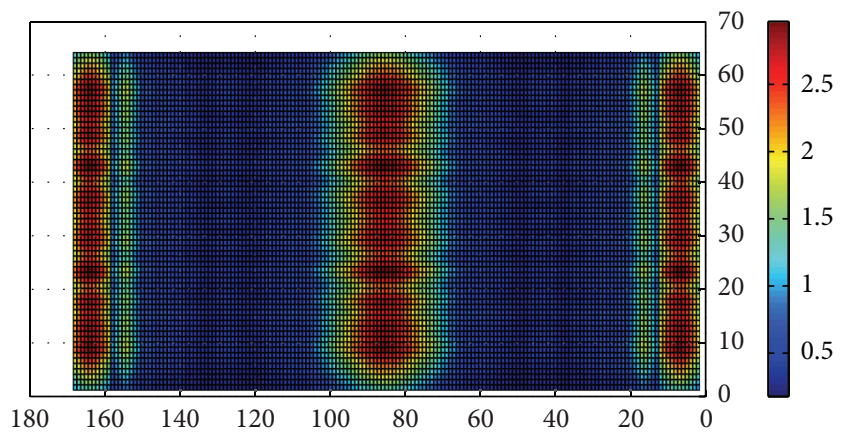

(c)

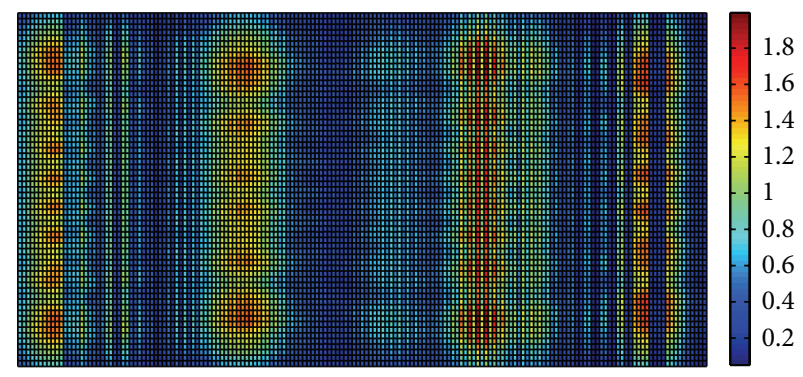

(b)

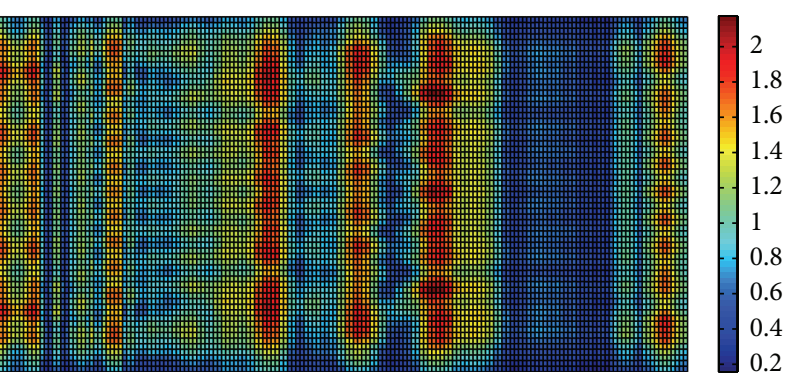

(d)

FIGURE 9: Simulation results of ultrasound distribution for cylindrical structure and arrays with different arrangement: (a) cylindrical transducer, (b) array in triangular arrangement, (c) array in quadrangular arrangement, and (d) array in pentagonal arrangement. When the color changes from blue to red, the vibration amplitude of a point increases.

choice for applications with a requirement of higher ultrasound generation efficiency. Compared with other transducer array applications which use arrays for higher resolution or performance in detection, the focus on higher energy efficiency in this paper is relatively novel as well. Future works can be focused on the design and implementation of the universal driving circuit for the transducer arrays.

\section{Conflict of Interests}

The author declares that there is no conflict of interests regarding the publication of this paper.

\section{References}

[1] A. S. Badday, A. Z. Abdullah, K. T. Lee, and M. S. Khayoon, "Intensification of biodiesel production via ultrasonic-assisted process: a critical review on fundamentals and recent development," Renewable and Sustainable Energy Reviews, vol. 16, no. 7, pp. 4574-4587, 2012.

[2] K. Ramachandran, T. Suganya, N. Gandhi, and S. Renganathan, "Recent developments for biodiesel production by ultrasonic assist transesterification using different heterogeneous catalyst: a review," Renewable \& Sustainable Energy Reviews, vol. 22, pp. 410-418, 2013.

[3] J. Luo, Z. Fang, and R. L. Smith Jr., "Ultrasound-enhanced conversion of biomass to biofuels," Progress in Energy and Combustion Science, vol. 41, no. 1, pp. 56-93, 2014.
[4] N. A. Tsochatzidis, P. Guiraud, A. M. Wilhelm, and H. Delmas, "Determination of velocity, size and concentration of ultrasonic cavitation bubbles by the phase-Doppler technique," Chemical Engineering Science, vol. 56, no. 5, pp. 1831-1840, 2001.

[5] M. H. Entezari and P. Kruus, "Effect of frequency on sonochemical reactions II. Temperature and intensity effects," Ultrasonics Sonochemistry, vol. 3, no. 1, pp. 19-24, 1996.

[6] S. Merouani, O. Hamdaoui, Y. Rezgui, and M. Guemini, "Effects of ultrasound frequency and acoustic amplitude on the size of sonochemically active bubbles-theoretical study," Ultrasonics Sonochemistry, vol. 20, no. 3, pp. 815-819, 2013.

[7] M. Hauptmann, H. Struyf, P. Mertens et al., "Towards an understanding and control of cavitation activity in $1 \mathrm{MHz}$ ultrasound fields," Ultrasonics Sonochemistry, vol. 20, no. 1, pp. 77-88, 2013.

[8] A. Iskalieva, B. M. Yimmou, P. R. Gogate, M. Horvath, P. G. Horvath, and L. Csoka, "Cavitation assisted delignification of wheat straw: a review," Ultrasonics Sonochemistry, vol. 19, no. 5, pp. 984-993, 2012.

[9] Wikipedia, "Mechanism of sonoluminescence," 2014, http://en .wikipedia.org/wiki/Mechanism_of_sonoluminescence.

[10] P. R. Gogate, A. M. Wilhelm, and A. B. Pandit, "Some aspects of the design of sonochemical reactors," Ultrasonics Sonochemistry, vol. 10, no. 6, pp. 325-330, 2003.

[11] S. Merouani, O. Hamdaoui, Y. Rezgui, and M. Guemini, "Energy analysis during acoustic bubble oscillations: relationship between bubble energy and sonochemical parameters," Ultrasonics, vol. 54, no. 1, pp. 227-232, 2014. 
[12] S. Koda, T. Kimura, T. Kondo, and H. Mitome, "A standard method to calibrate sonochemical efficiency of an individual reaction system," Ultrasonics Sonochemistry, vol. 10, no. 3, pp. 149-156, 2003.

[13] L. Csoka, S. N. Katekhaye, and P. R. Gogate, "Comparison of cavitational activity in different configurations of sonochemical reactors using model reaction supported with theoretical simulations," Chemical Engineering Journal, vol. 178, pp. 384-390, 2011.

[14] T. Makuta, Y. Aizawa, and R. Suzuki, "Sonochemical reaction with microbubbles generated by hollow ultrasonic horn," Ultrasonics Sonochemistry, vol. 20, no. 4, pp. 997-1001, 2013.

[15] Y. Asakura, T. Nishida, T. Matsuoka, and S. Koda, "Effects of ultrasonic frequency and liquid height on sonochemical efficiency of large-scale sonochemical reactors," Ultrasonics Sonochemistry, vol. 15, no. 3, pp. 244-250, 2008.

[16] Y. W. Yang, L. Zhang, Z. L. Jin, and C. K. Soh, "Smart plates and shells," in Smart Materials in Structural Health Monitoring, Control and Biomechanics, Advanced Topics in Science and Technology in China, pp. 333-393, Springer, Berlin, Germany, 2012.

[17] C. M. Rumack, S. R. Wilson, and J. William Charboneau, Diagnostic Ultrasound, vol. 1, Mosby-Year Book, St. Louis, Mo, USA, 1991. 


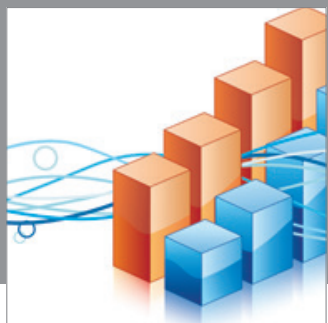

Advances in

Operations Research

mansans

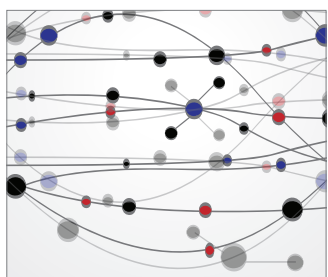

The Scientific World Journal
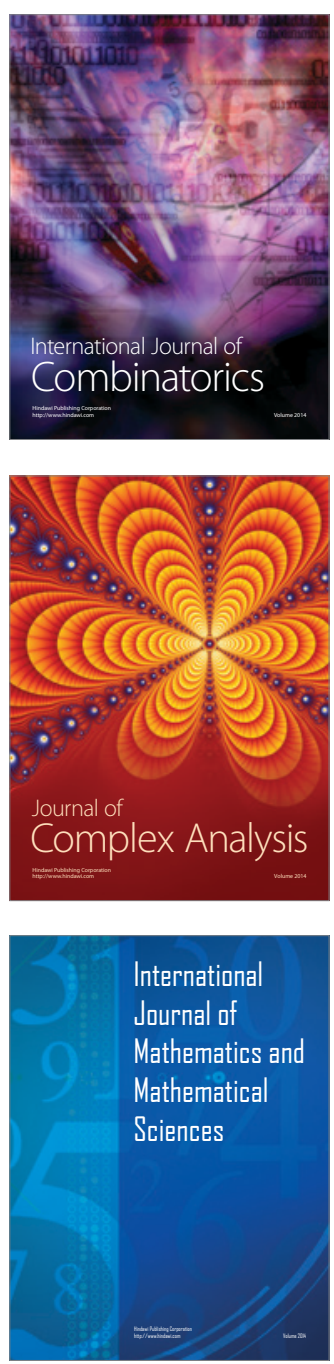
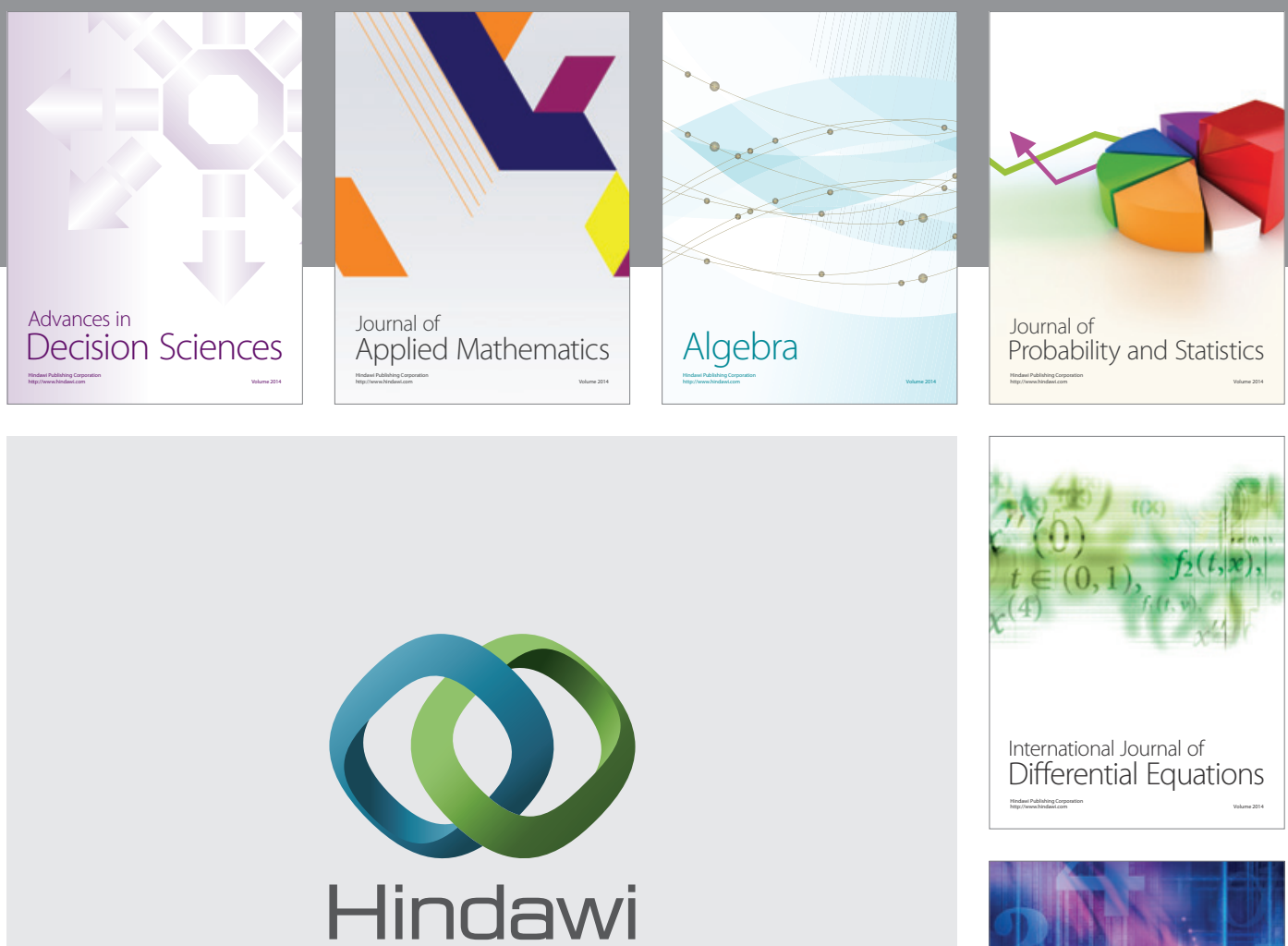

Submit your manuscripts at http://www.hindawi.com
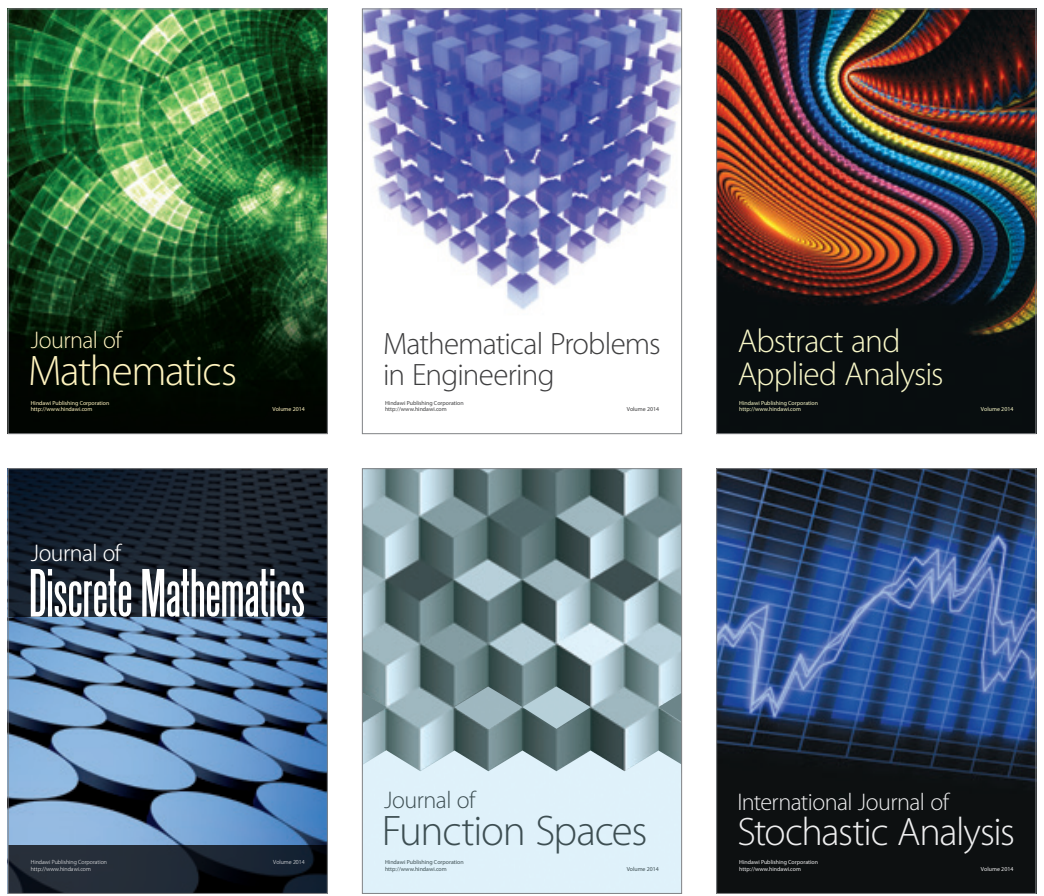

Journal of

Function Spaces

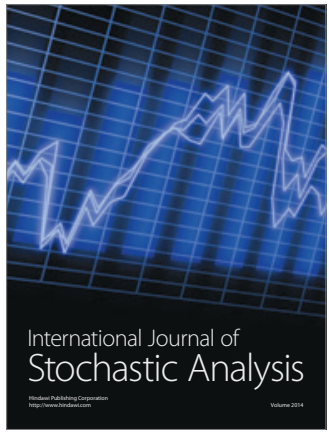

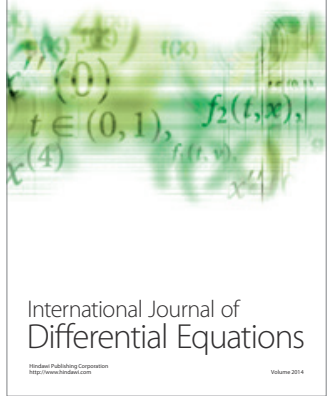
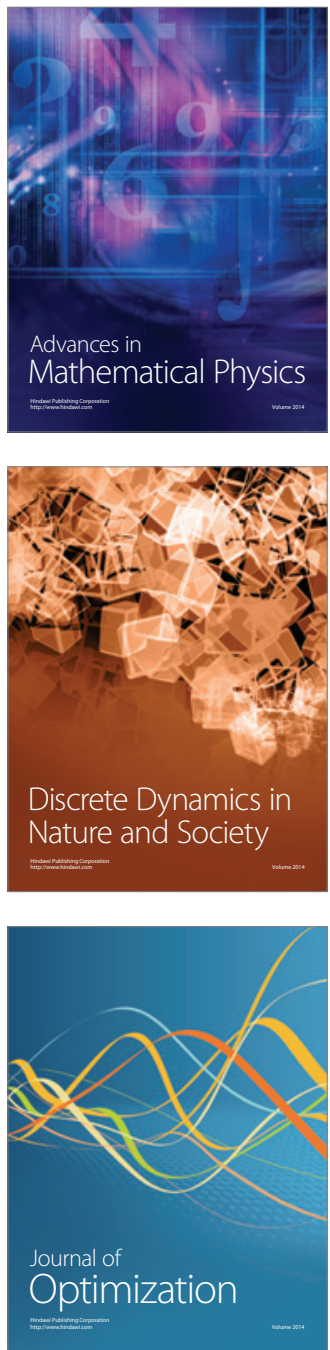